\section{Vorhersagemodelle für Suizidversuche und Suizide entwickelt}

Simon GE et al. Predicting Suicide Attempts and Suicide Deaths Following Outpatient Visits Using Electronic Health Records. Am J Psychiatry 2018; 175: 951-960. doi:10.1176/appi.ajp.2018.17101167

In den USA war zwischen 2000 und 2016 ein Anstieg der Suizidraten um $25 \%$ zu verzeichnen. Die Hälfte der Personen, die Suizid begehen, erhielten im Jahr davor eine Diagnose hinsichtlich einer seelischen Erkrankung oder eine entsprechende Behandlung. G. E. Simon et al. haben auf Basis von elektronischen Gesundheitsakten Modelle zur Vorhersage von Selbstmordversuchen oder Selbstmorden nach einem ambulanten Besuch entwickelt und validiert.

Die Studie berücksichtigte 7 Gesundheitssysteme mit insgesamt 2960929 Patienten. Lediglich Individuen mit einem Mindestalter von 13 Jahren gingen in die Analyse ein. Für die Auswertung lagen Daten von 10275853 Besuchen in einer Fachklinik für psychische Erkrankungen vor und von 9685206 Besuchen, bei denen die Diagnosen zur psychischen Gesundheit im Rahmen der Primärversorgung erstellt worden waren. Die Autoren konzentrierten sich dabei auf die Zeitspanne zwischen 1. Januar 2009 und 30. Juni 2015. Als mögliche Prädiktoren für Selbstmordversuche und Selbstmorde wurden 313 demografische und klinische Charakteristika aus den Gesundheitsakten extrahiert, wobei Zeiträume von bis zu 5 Jahren vor jedem Besuch evaluiert wurden. Dazu gehörten beispielsweise vorangegangene seelische Diagnosen und Medikamenteneinnahmen, Suizidversuche, Verletzungen bzw. Vergiftungen in der Vergangenheit oder ein Krankenhausaufenthalt bzw. eine Notfallaufnahme aufgrund psychischer Ursachen sowie Ergebnisse von routinemäßig eingesetzten Fragebögen zu Depressionen.

\section{Ergebnisse}

Das Durchschnittsalter der Patienten betrug 46 Jahre, $62 \%$ waren weiblich.
Die Auswertung der vorhandenen Datenquellen erbrachte 24133 Suizidversuche und 1240 Suizide innerhalb von 90 Tagen nach einem Arztbesuch. Als stärkste Prädiktoren für Selbstmordversuche erwiesen sich sowohl bei Fachklinikpatienten als auch bei solchen aus der Primärversorgung ein früherer Suizidversuch, seelische Diagnosen und Medikamenteneinnahmen, ein Bejahen von Punkt 9 des Fragebogens PHQ-9 sowie ein Klinikaufenthalt oder eine Notfallaufnahme aus psychischen Gründen. Die Datenauswertung bei Patienten, die Fachkliniken besucht hatten, ergab folgendes: Für $43 \%$ aller nachfolgenden Suizidversuche und $48 \%$ der Suizide waren Besuche mit Risiko-Scores im oberen 5\%-Bereich verantwortlich. Unter den Patienten aus diesem 5\%Bereich begingen innerhalb von 90 Tagen 5,4\% einen Suizidversuch und $0,26 \%$ Suizid. Die "C-Statistics“, die der "Area under the Curve" entsprechen, für die Vorhersage eines Selbstmordversuches oder eines durchgeführten Selbstmordes betrugen jeweils 0,851 und 0,861. Die Ergebnisse für Patienten aus dem Grundversorgungsbereich stellten sich wie folgt dar: Besuche mit Risiko-Scores im Top- 5\%-Bereich machten hier $48 \%$ der Suizidversuche und $43 \%$ der tatsächlichen Suizide aus. Die Ergebnisse der "C-Statistics" waren jeweils 0,853 und 0,833 .

\section{FAZIT}

Laut den Studienautoren sind Vorhersagemodelle, die Ergebnisse aus Gesundheitsakten und Selbstbeurteilungsfragebögen berücksichtigen, vorhandenen Methoden zur Vorhersage des Selbstmordrisikos überlegen. Allerdings seien solche Vorhersagemodelle nicht in der Lage, eine klinische Beurteilung zu ersetzen. Risiko-Scores könnten aber bei der individuellen klinischen Entscheidungsfindung sowie innerhalb von Programmen zur Qualitätsverbesserung hilfreich sein.

Dr. Frank Lichert, Weilburg 